\title{
Case - Renal artery pseudoaneurysm: A life-threatening occurrence following thulium laser lithotripsy
}

Ajit Monteiro, $\mathrm{MD}^{1}$; Kyle M. Waisanen, $\mathrm{MD}^{2}$; Eugene V. Ermolovich, MD²; Ichabod S. Jung ${ }^{2,3}$; John M. Roehmholdt ${ }^{2,3}$

${ }^{1}$ Department of Pediatrics, University at Buffalo, Clinical and Translational Research Center, Buffalo, NY, United States; ${ }^{2}$ Department of Urology, University at Buffalo, Jacobs School of Medicine and Biomedical Sciences, Buffalo, NY, United States; ${ }^{3}$ Western New York Urology Associates, Cheektowaga, NY, United States

Cite as: Monteiro A, Waisanen KM, Ermolovich EV, et al. Case - Renal artery pseudoaneurysm: A life-threatening occurrence following thulium laser lithotripsy. Can Urol Assoc J 2021 Deceber 21; Epub ahead of print. http://dx.doi.org/10.5489/cuaj.7548

Published online December 21, 2021

Corresponding author: Dr. Ajit Monteiro, Department of Pediatrics, Buffalo, NY, United States; ajit.monteiro93@gmail.com

$* * *$

\section{Introduction}

Renal artery pseudoaneurysm post-laser lithotripsy is a rare and potentially life-threatening complication. Traditionally, the most common modalities used for treatment of urinary tract stones were holmium laser lithotripsy and extracorporeal shockwave lithotripsy. However, thulium laser has recently gained momentum in the treatment of urinary tract stones with increasing use and availability in healthcare systems. We report a case of renal artery pseudoaneurysm post-thulium laser lithotripsy who presented in hemorrhagic shock after ureteral stent removal and was subsequently stabilized by endovascular embolization.

\section{Case report}

A 24-year-old male with past medical history of recurrent renal calculi presented with left $6 \mathrm{~mm}$ renal pelvis and $7 \mathrm{~mm}$ upper pole calculi which was diagnosed on Computerized topography (CT) abdomen and pelvis. He underwent flexible ureteroscopy with thulium laser lithotripsy and double $\mathrm{J}$ ureteral stent insertion. The procedure was uneventful with institutional standard pulsed thulium soft stone laser settings of $0.4 \mathrm{~J}$ at $40 \mathrm{~Hz}$ totalling $16 \mathrm{w}$ of power and hard stone settings of $2 \mathrm{~J}$ at $20 \mathrm{~Hz}$ totalling $40 \mathrm{w}$ of power for duration of procedure. The patient was discharged home from ambulatory setting the same day with ureteral stent in-situ and planned outpatient stent removal. On post-operative day \#20, the patient presented to the emergency department with haematuria since prior night associated with penile pain, tremors, cold sweats. CT abdomen/pelvis with IV contrast showed moderate amount of amorphous intermediate density present in the lumen of the urinary bladder which likely represented the presence of blood or blood degradation products. The left ureteral stent was visible in appropriate anatomic positioning and without associated hydronephrosis. The pain and cold sweats subsided with spontaneous passage of the urinary clot in the ED. His vitals remained unremarkable and 
laboratory testing exhibited: Hgb $6.45 \mathrm{mmol} / \mathrm{L}$, WBC 18.8, UA (3+ Blood, Nitrite +, Leukocyte Esterase 2+, WBC 26-100, RBC >100, Moderate Bacteria). He was given 1 gram of intravenous ceftriaxone and discharged on oral Bactrim for 7 days to treat for a presumed urinary tract infection. The next day, he underwent cystoscopy for stent removal which yielded the presence of a small volume retained urinary clot in the bladder lumen originating from the left ureteral orifice. Post-operative day \#2 after stent removal, the patient presented to the ED with syncopal episodes, gross bright red haematuria, passage of large urinary clots, peripheral numbness and tingling, palpitations, and chills. Vital signs were consistent with stage 4 haemorrhagic shock which was confirmed with $\mathrm{Hgb} 4.65 \mathrm{mmol} / \mathrm{L}$ from pre-operative $8.75 \mathrm{mmol} / \mathrm{L}$ and accompanying metabolic acidosis. Tranexamic Acid (TXA) was administered along with two units of packed red blood cells with continued worsening of mentation and unstable vital signs. CT angiography of the torso was performed revealing a $10 \mathrm{~mm}$ x $7 \mathrm{~mm}$ left renal artery pseudoaneurysm arising from an early branching segment. He was emergently transferred to the interventional radiology suite and under-going selective renal artery embolization. Postembolization, the patient was then transferred to the intensive care unit having received a total of five PRBCs, TXA, and 3 liters of crystalloid intravenous fluids for resuscitation. The patient was discharged from the hospital on day three with well controlled left flank pain on oral hydrocodone acetaminophen 5/325 mg, no further haematuria and stable $\mathrm{Hgb}$ of $6.02 \mathrm{mmol} / \mathrm{L}$. Repeat CT abdomen/pelvis on morning of discharge found no persistence of the previously identified pseudoaneurysm or vascular contrast extravasation and only expectant thrombus of a subsegmental left renal artery, with infarct and stable embolization.

\section{Discussion}

Renal artery pseudoaneurysms are an exceedingly rare vascular lesion that occurs due to damage of the arterial wall structure with locally contained hematoma that is covered by coagulated and fibrous tissue ${ }^{1,2}$. Renal artery pseudoaneurysms may also be marginally more common than reported as CT with contrast is not routinely performed following the majority of endoscopic urologic procedures ${ }^{1}$. Complications can include thromboembolism, neurovascular compression, infection and life-threatening rupture with exsanguination. While the risk of rupture is low, it is associated with high mortality rates reaching $80 \%$ in reported cases and the risk of bleeding increases if the size of the pseudoaneurysm $>2 \mathrm{~cm}^{3}$. Renal artery pseudoaneurysms have been reported in various clinical scenarios, including blunt renal trauma, surgery, percutaneous procedures, and spontaneously secondary to inflammatory or neoplastic processes within the kidney ${ }^{4}$. Patients typically present with flank pain, haematuria or unexplained anemia, although many can be asymptomatic. In severe cases, these aneurysms may rupture and lead to hemorrhagic shock warranting emergent intervention for survival.

The diagnosis of pseudoaneurysm in this case was made with CT angiography performed on post-operative day \#22 prior to proceeding with intravascular embolization for definitive treatment. On further retrospective analysis of imaging performed during the patient's first ED presentation, a subtle dilation of the lower pole subsegmental renal artery at the site of the later diagnosed pseudoaneurysm could be seen however incompletely imaged during an insufficient arterial phase on CT imaging. Earlier detection of this vascular injury would have aided in prompt treatment of the injury decreasing the risk of life-threatening haemorrhage seen in this 
case. A major factor which may have increased the risk of pseudoaneurysm in our patient was the use of high energy settings for renal stone fragmentation. The use of $40 \mathrm{w}$ energy output fragmentation when paired with a renal pelvis stone location allows for a thin barrier of tissue between the laser and abutting posterior renal hilar vasculature. Contemporary recommendations for thulium laser lithotripsy settings range from $15-30 \mathrm{w}$ within the renal collecting system, 10$15 \mathrm{w}$ within the ureter, and 30-50 w within the bladder lumen ${ }^{5}$. Lower optimized power recommendations are further echoed by manufacturer recommendations of total power outputs no greater than $25 \mathrm{w}$ for renal calculi and $20 \mathrm{w}$ for ureteral calculi. Thulium laser has recently been gaining momentum in the treatment of renal stones ${ }^{6,7}$. This laser has been proven to be effective as they emit a more well distributed pulsed laser waves resulting in rapid stone ablation and lower retropulsion in comparison to holmium laser ${ }^{8,9}$. We performed a thorough search of contemporary publication databases and failed to find any reports describing renal artery pseudoaneurysms after thulium laser lithotripsy.

Renal artery pseudoaneurysms can be managed by either open surgical or endovascular access, depending on the stability of the patient ${ }^{10}$. Endovascular selective angioembolization is the mainstay of treatment, although open surgical correction might be indicated if unable to obtain haemostasis endovascular techniques or repair of the arterial wall defect are required ${ }^{11}$. For patients with contraindication to the use of contrast or complex vascular anatomy including numerous intraluminal calcifications, percutaneous ultrasound-guided embolization may be used $^{2}$. Open surgical ligation, endovascular stent, and intra-aneurysmal thrombin with balloon occlusion have also been described as alternative treatment options. Further literature has described spontaneous resolution of pseudoaneurysms after continued observation, however due to the difficulty in accurately predicting the outcome, it is best to intervene ${ }^{2,12}$.

\section{Conclusions}

A low threshold of suspicion for a renal vascular injuries such as a pseudoaneurysm should be maintained when a patient presents with profuse gross haematuria post endoscopic urological interventions utilizing energy devices. The recognition of this injury as a most-probable diagnosis and early vascular control with endovascular intervention may prevent potential lifethreatening haemorrhage as described in this case with a vascular injury known for high mortality rates. 


\section{References}

1. Jubber I, Patel PR, Hori S, Al-Hayek S. Renal pseudoaneurysm: a rare and potentially fatal complication following ureteroscopy and laser fragmentation of stones. Annals of the Royal College of Surgeons of England. 2018 Mar;100(3):e51-2.

2. Ngo TC, Lee JJ, Gonzalgo ML. Renal pseudoaneurysm: an overview. Nature Reviews Urology. 2010 Nov;7(11):619-25.

3. Hadjipavlou M, Abbaraju J, Serafimov V, Madaan S. Spontaneous rupture of a renal artery pseudoaneurysm with no precipitating risk factor. JRSM open. 2018 Apr;9(4):2054270418758568.

4. Durner L, El Howairis MEF, Buchholz N. Renal Pseudoaneurysm after Flexible Ureterorenoscopy - An Unusual Complication. Urol Int [Internet]. 2017 [cited 2020 Oct 10];99(4):484-6. Available from: https://www.karger.com/Article/FullText/441042

5. Kronenberg, P. and O. Traxer (2019). "The laser of the future: reality and expectations about the new thulium fiber laser-a systematic review." Transl Androl Urol 8(Suppl 4): S398-S417.

6. Traxer O, Keller EX. Thulium fiber laser: the new player for kidney stone treatment? A comparison with Holmium:YAG laser. World Journal of Urology. 2020 Aug;38(8):188394.

7. Hall LA, Gonzalez DA, Fried NM. Thulium fiber laser ablation of kidney stones using an automated, vibrating fiber. J Biomed Opt. 2019;24(3):1-10.

8. Zhang F, Shao Q, Herrmann TRW, Tian Y, Zhang Y. Thulium laser versus holmium laser transurethral enucleation of the prostate: 18-month follow-up data of a single center. Urology. 2012 Apr;79(4):869-74.

9. Andreeva V, Vinarov A, Yaroslavsky I, Kovalenko A, Vybornov A, Rapoport L, et al. Preclinical comparison of superpulse thulium fiber laser and a holmium:YAG laser for lithotripsy. World Journal of Urology. 2020 Feb;38(2):497-503.

10. Roman LI, Feel CF, França VT, Merten CM, Dummer CD. Renal artery pseudoaneurysm. Jornal Brasileiro de Nefrologia [Internet]. 2017 [cited 2020 Oct 10];39(4). Available from:

http://www.scielo.br/scielo.php?script=sci_arttext\&pid=S0101-28002017000400458

11. Inci K, Cil B, Yazici S, Peynircioglu B, Tan B, Sahin A, et al. Renal artery pseudoaneurysm: complication of minimally invasive kidney surgery. Journal of Endourology. 2010 Jan;24(1):149-54.

12. Guyot R, Arnoux V, Descotes J-L, Terrier N, Boillot B, Thuillier C, et al. Prise en charge des pseudo-anévrismes rénaux intraparenchymateux post-traumatiques : à propos d'une série de 325 patients traumatisés rénaux. Progrès en Urologie [Internet]. 2017 Mar [cited 2020 Oct 10];27(3):190-9. Available from:

https://linkinghub.elsevier.com/retrieve/pii/S1166708716307849 


\section{Figures and Tables}

Fig 1. Left: CTA torso showing the left renal artery pseudoaneurysm. Right: The left renal artery pseudoaneurysm with injection of contrast.

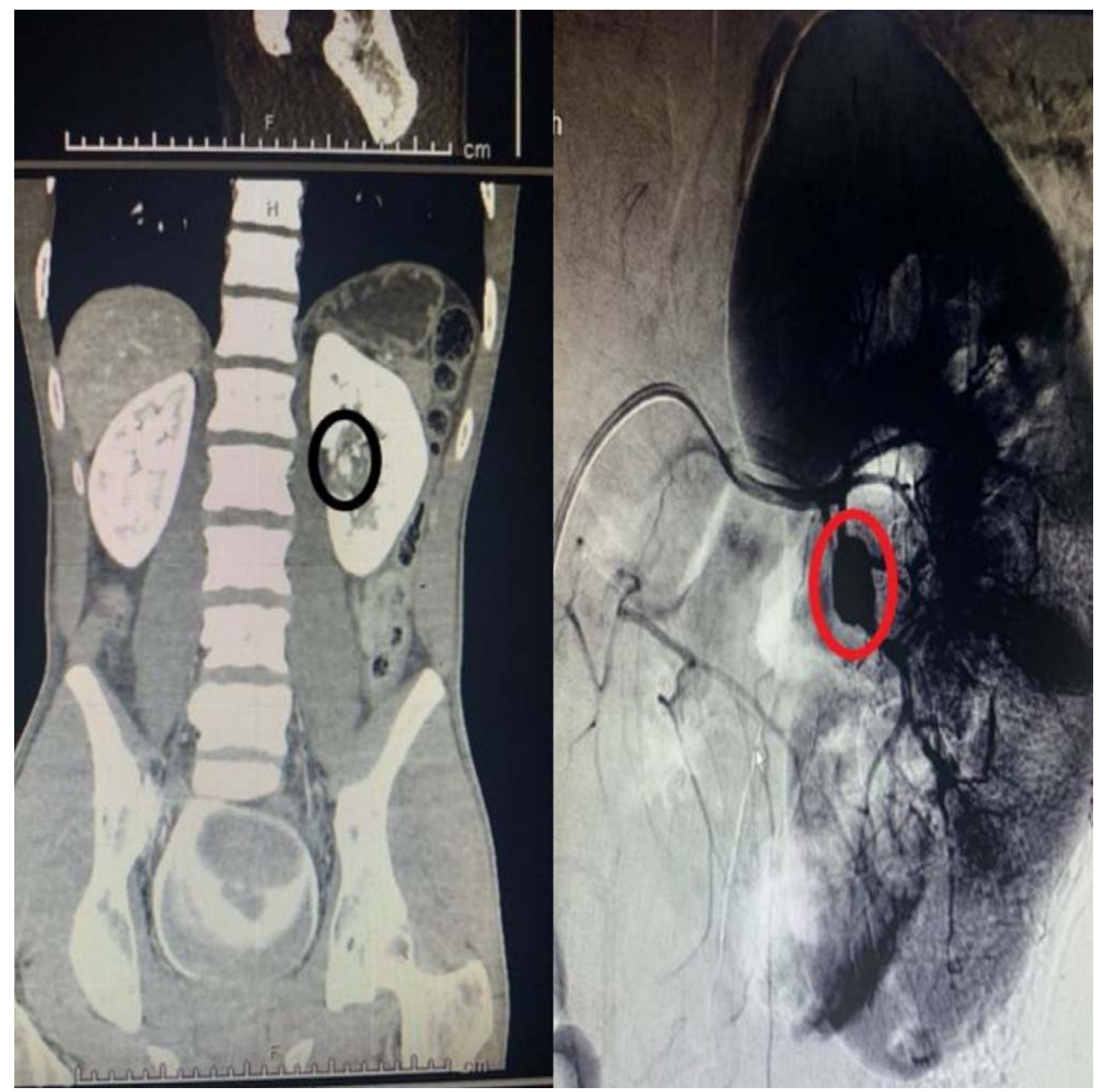


Fig 2. Left: $\mathrm{CT} A B D / P E L$ from POD\#20 showing possible vascular defect though not diagnosed. Right: CTA ABD/PEL from POD\#22 with renal artery pseudoaneurysm.

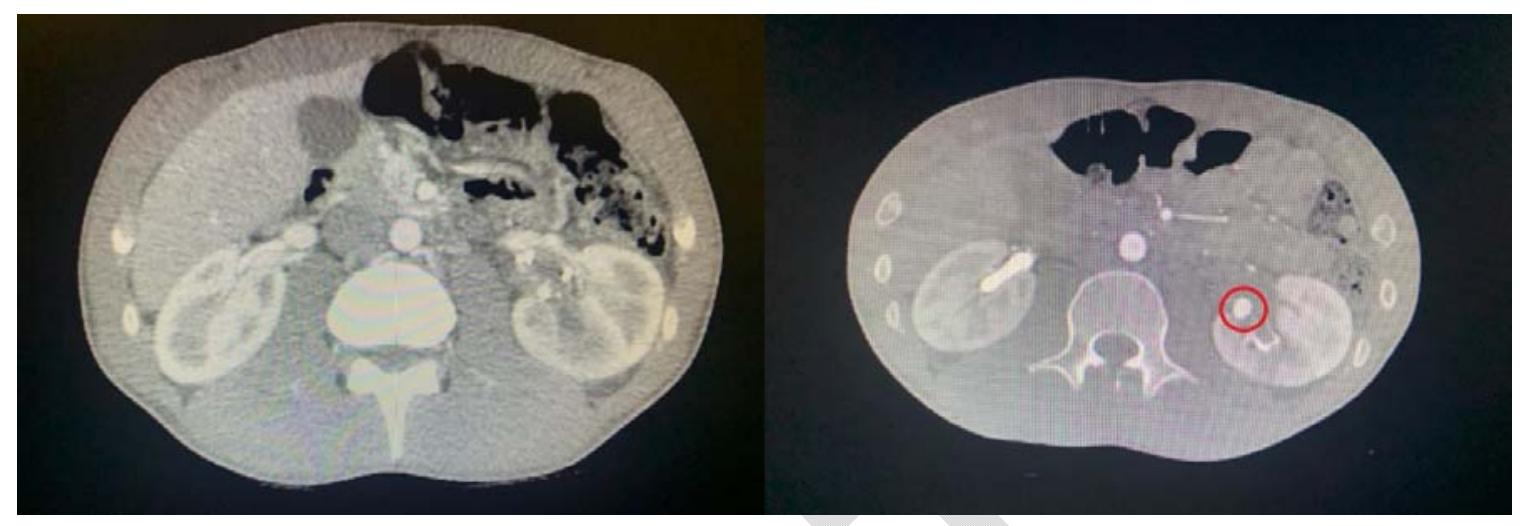

